\title{
Procyanidins mediates antineoplastic effects against non-small cell lung cancer via the JAK2/STAT3 pathway
}

\author{
Yue $\mathrm{Wu}^{1,2 \#}$, Chi Liu ${ }^{1 \#}$, Yuxu Niu ${ }^{1,2 \#}$, Jiamin $\mathrm{Xia}^{3}$, Liwen Fan ${ }^{1,2}$, Yun $\mathrm{Wu}^{1,2}$, Wen Gao ${ }^{1,2}$ \\ ${ }^{1}$ Shanghai Key Laboratory of Clinical Geriatric Medicine, Huadong Hospital, Fudan University, Shanghai, China; ${ }^{2}$ Department of Thoracic Surgery, \\ Huadong Hospital, Fudan University, Shanghai, China; ${ }^{3}$ Ministry of Education, School of Public Health, Tongji Medical College, Huazhong \\ University of Science and Technology, Wuhan, China \\ Contributions: (I) Conception and design: Y Wu, C Liu; (II) Administrative support: Y Wu, W Gao; (III) Provision of study materials or patients: Y \\ Wu, C Liu, J Xia; (IV) Collection and assembly of data: Y Wu, Y Niu, L Fan; (V) Data analysis and interpretation: Y Wu, C Liu; (VI) Manuscript \\ writing: All authors; (VII) Final approval of manuscript: All authors. \\ "These authors contributed equally to this work. \\ Correspondence to: Yun Wu; Wen Gao. Department of Thoracic Surgery, Huadong Hospital Affiliated to Fudan University, No. 221 West Yanan \\ Road, Shanghai 200040, China. Email: a.oret@live.cn; gaowenchest@163.com.
}

Background: Lung cancer is a malignant tumor with one of the highest rates of cancer-related morbidity and mortality worldwide. Non-small cell lung cancer (NSCLC) account for $85 \%$ of all lung cancers and have a poor prognosis. Proanthocyanidins (PCs) are polyphenolic compounds that are found widely in natural plants. The present study aimed to determine the effects of PC on lung cancer and identify its possible mechanism.

Methods: A cell growth assay was used to detect the cell growth ability of A549 cancer cells, and a clonal formation assay was used to detect the cloning ability of A549 cancer cells. Flow cytometry was used to detect the effect of PCs on apoptosis and the cell cycle. The wound healing test, Transwell migration, and invasion test were used to detect the migration and invasion of human NSCLC A549 cells. Western blotting was utilized to detect the expression levels of N-cadherin, E-cadherin, vimentin, Janus kinase 2 (JAK2), p-signal transducer and activator of transcription 3 (p-STAT3), STAT3, matrix metalloproteinase 2 (MMP-2), MMP-9, and the apoptosis-related proteins, B-cell lymphoma-2 (Bcl-2) and BCL2-associated X (Bax). Cell immunofluorescence was used to detect the expression levels of the p-STAT3 primary antibody.

Results: PCs reduced the proliferation and cloning ability of A549 cells and significantly inhibited the migration and invasion of A549 cells in a dose-dependent manner. At the same time, PCs induced apoptosis in A549 cells and G2/M cell cycle arrest. PCs increased the pro-apoptotic protein expression, Bax, and down-regulated the anti-apoptotic protein expression, Bcl-2. PCs also inhibited the epithelial-mesothermal transition (EMT) process of A549 cells. We also found that the JAK2/STAT3 signaling pathway inhibitor, AG490, cooperated with PCs to inhibit A549 cell invasion and migration. Our results demonstrated that PCs could mediate the antitumor effect of NSCLC via the JAK2/STAT3 pathway.

Conclusions: PCs can inhibit NSCLC A549 cell proliferation, invasion, metastasis, clone formation, EMT, and induced apoptosis and G2/M cell cycle arrest. They work by inhibiting the JAK2/STAT3 signaling pathway. As a novel antitumor drug, PCs have broad application prospects for the treatment of NSCLC.

Keywords: Proanthocyanidins; non-small cell lung cancer (NSCLC); Janus kinase 2/signal transducer and activator of transcription 3 (JAK2/STAT3); epithelial-mesothermal transition (EMT)

Submitted Oct 09, 2020. Accepted for publication Apr 09, 2021.

doi: $10.21037 /$ tcr-20-3018

View this article at: http://dx.doi.org/10.21037/tcr-20-3018 


\section{Introduction}

Lung cancer is the leading cause of cancer-related deaths worldwide, with a 5-year survival rate (after diagnosis) of only $17.7 \%(1,2)$. Non-small cell lung cancer (NSCLC) accounts for $85 \%$ of all lung cancers (1). Currently, the pharmacological effects of proanthocyanidins (PCs) have been confirmed, and there are few side effects.

PCs (Figure 1A) are polyphenolic compounds composed of dimers or polymers of catechins and epicatechins. They are natural compounds with special biological activity that are common in plants such as blueberries and grape seeds $(3,4)$. A large number of studies have found that glucoproanthocyanidins have significant anti-tumor effects, in addition to scavenging free radicals and antiinflammatory effects $(5,6)$. In recent years, the antitumor effect of PCs has attracted the attention of researchers. Previous studies have shown that PCs have antitumor effects on various cancers, including esophageal, liver, lung, and prostate cancers (7-10). Existing research has also demonstrated that PCs may exert antitumor effects by inhibiting lung cancer cell invasion, migration, and cell proliferation. However, the specific mechanism of action remains unclear (10).

Through this study, we found for the first time that PCs can induce G2/M cell cycle arrest and apoptosis of lung cancer cells. They can effectively inhibit the proliferation, invasion, migration, and epithelial-mesothermal transition (EMT) of lung cancer A549 cells via the Janus kinase 2 (JAK2)/signal transducer and activator of transcription 3 (STAT3) pathway and induce A549 cell G2/M cell cycle arrest and apoptosis. We present the following article in accordance with the MDAR reporting checklist (available at http://dx.doi.org/10.21037/tcr-20-3018).

\section{Methods}

\section{Cell culture}

Human NSCLC cell lines A549 were obtained from the Cell Bank of the Chinese Academy of Science (Shanghai, China). The cells were cultured in Roswell Park Memorial Institute (RPMI)-1640 medium (Gibco; Thermo Fisher Scientific, lnc.) containing 10\% fetal bovine serum (FBS) (Gemini Bio, California, USA) $100 \mathrm{U} / \mathrm{mL}$ penicillin, and $100 \mu \mathrm{g} / \mathrm{mL}$ streptomycin in $5 \% \mathrm{CO}_{2}$ at $37^{\circ} \mathrm{C}$.

\section{Cell growth assay}

A cell growth assay was utilized to detect the effect of PCs on the proliferation of A549 cells. The cells were counted under a microscope (Nikon, Japan), and the cell concentration was adjusted to approximately $5 \times 10^{4}$ cells/ $\mathrm{mL}$. Next, the cells were inoculated in a 96-well plate (Corning Costar, New York, NY, USA) for culture, and 100 $\mu \mathrm{L}$ of cell suspension was added to each well (approximately 5,000 cells). A549 cells were pretreated with $0.1 \%$ dimethyl sulfoxide (DMSO) (control group) and 12.5, 25, 50, 100, $200 \mu \mathrm{M}$ procyanidins (X-Y Biotechnology, Shanghai, China) for 24 and 48 hours, respectively.

Following the treatment, the Cell Counting Kit-8 (CCK-8) reagent (Beyotime, Shanghai, China) was added to the RPMI-1640 medium and mixed uniformly at 1:10 as the reaction solution, and $10 \mu \mathrm{L}$ of CCK-8 reagent was added to each well to be tested. The reaction solution was added to the 96-well plate and placed in an incubator at $37^{\circ} \mathrm{C}$ and $5 \% \mathrm{CO}_{2}$. After approximately 2 hours, color development was observed and was immediately tested on the Synergy H1 microplate reader (Bio-Rad Laboratories, Hercules, CA, USA). During the detection, the wavelength of the Synergy $\mathrm{H} 1$ microplate reader was adjusted to $450 \mathrm{~nm}$, and the relative content of the cells in the wells was analyzed by detecting the absorbance of each well. This experiment was repeated three times.

\section{Clonal formation assay}

A549 cells (1,000 cells/well) were seeded into a six-well plate and placed in a $37{ }^{\circ} \mathrm{C}, 5 \% \mathrm{CO}_{2}$ incubator overnight. Next, 0.1\% DMSO (control), procyanidins (25, 50, $100 \mu \mathrm{M})$ were co-cultured with A549 cells for 7 days. The RPMI-1640 medium (containing 10\% FBS) was replaced every 2 days. After 7 days of incubation, the sixwell plate was removed, and the cells were fixed with $4 \%$ paraformaldehyde (Beyotime) and stained with crystal violet solution (Beyotime). The number of colonies formed by $>50$ single cells was then counted. Three independent experiments were conducted.

\section{Wound bealing assay}

A wound-healing assay was employed to detect the effect of PCs on the migration of A549 cells. A549 cells were seeded on 
a six-well plate (Beyotime) at a density of $5.0 \times 10^{6}$ cells/well, and incubated in a $37^{\circ} \mathrm{C}, 5 \% \mathrm{CO}_{2}$ incubator. It is important to note that when culturing to more than $90 \%$ cell confluence, the medium should be aspirated, and a sterile $200 \mu \mathrm{L}$ pipette tip used to divide the cell layer, creating a cell-free gap between two adjacent areas. Subsequently, $0.1 \%$ DMSO (control) and 25, 50, $100 \mu \mathrm{M}$ procyanidins were co-cultured with A549 cells for 48 hours. Each well was rinsed gently with phosphate buffer saline (PBS) three times before taking a photograph. Next, a serum-free medium was added, and a random photograph of the area of migrating cells at three different locations along the scratch length was taken at 0,24 , and 48 hours. According to the following formula, the wound closure rate was calculated: wound closure rate $(\%)=(\mathrm{Ai}-\mathrm{Af}) / \mathrm{Ai} \times 100 \%$, where $\mathrm{Ai}$ is the wound scratch area at the initial time, and Af is the wound healing area at the final time. This experiment was repeated three times.

\section{Transwell migration and invasion assays}

Transwell migration and invasion assays were used to detect the effect of PCs on the migration and invasion of A549 cells. An $8 \mu \mathrm{m}$ pore size Transwell cell (Corning Costar, New York, NY, USA) was used for cell migration and invasion assays. Matrigel (BD, San Jose, CA, USA) was precoated in the upper chamber, and A549 cells $\left(5 \times 10^{4}\right.$ cells/ well) were suspended in $200 \mu \mathrm{L}$ serum-free RPMI-1640 medium were seeded into the upper chamber. In the lower chamber, $600 \mu \mathrm{L}$ of RPMI-1640 medium containing $10 \%$ FBS was added as a chemical attractant. The cells were then incubated in a $37^{\circ} \mathrm{C}, 5 \% \mathrm{CO}_{2}$ incubator for 12 hours, and the cells on the top surface were removed with a cotton swab. The infiltrating cells on the membrane's basal side were fixed with $4 \%$ paraformaldehyde and stained with $0.5 \%$ crystal violet (Sigma, USA). Photos were then taken, and cells were counted under an optical microscope (Olympus, Tokyo, Japan). A similar method was used to study cell migration by measuring Transwell chambers without Matrigel. This experiment was repeated three times.

\section{Flow cytometry analysis_cell cycle}

Flow cytometry analysis was employed to detect the effect of PCs on the cell cycle of A549 cells. The cells were treated with DMSO and procyanidins $(25,50,100 \mu \mathrm{M})$ for 24 hours. The trypsinized cells were washed with cold PBS, and the heart was centrifuged at 2,000 rpm for 5 minutes.
The cells were slowly recovered with $50 \mu \mathrm{L}$ of PBS, and then $950 \mu \mathrm{L}$ of $75 \%$ ethanol was added to fix the cells at $4{ }^{\circ} \mathrm{C}$ overnight. The fixed cells were washed with cold PBS, centrifuged at 2,000 $\mathrm{rpm}$ for 5 minutes, and incubated with $500 \mu \mathrm{L}$ propidium iodide (PI)/RNase staining buffer (BD Biosciences, Franklin Lakes, NJ, USA) for 15 minutes in the dark at room temperature. The above cells were detected by flow cytometry (BD-FACSAria II, Franklin Lakes, NJ, USA). The cell cycle was analyzed with ModfitLT (VeritySoftwareHouse, USA), and the experiment was repeated three times.

\section{Flow cytometry analysis-apoptosis}

Flow cytometry analysis was utilized to detect the effect of PCs on the cell apoptosis of A549 cells. After treatment with DMSO (control group) and PCs $(25,50,100 \mu M)$ for 24 hours, the cells were washed twice with PBS and resuspended in $100 \mu \mathrm{L}$ annexin- $\mathrm{V}$ binding buffer (BD Biosciences, Franklin Lakes, NJ, USA) at a concentration of $2 \times 10^{6}$ cells $/ \mathrm{mL}$. Subsequently, $5 \mu \mathrm{L}$ annexin-V-PE (BD Biosciences, CA, USA) and $5 \mu \mathrm{L}$ 7-ADD (BD Biosciences, CA, USA) were added and incubated in the dark at room temperature for 15 minutes. Binding buffer $(400 \mu \mathrm{L})$ was then added to each test tube, and quantitative analysis of annexin V-PE/7-ADD staining was performed by flow cytometry within 1 hour after staining. Western blot (WB) was used to detect the down-regulation of the antiapoptotic protein, $\mathrm{Bcl}-2$, and the pro-apoptotic protein, Bax. This experiment was repeated three times.

\section{WB analysis}

The total cell lysate was separated with Radio Immunoprecipitation Assay (RIPA) buffer (Beyotime, Shanghai, China), and the concentration was determined using the bicinchoninic acid (BCA) method. Ten $\mu \mathrm{L}$ of the total lysate was separated using $10 \%$ sodium dodecyl sulfate polyacrylamide gel electrophoresis (SDS-PAGE) and then transferred to a polyvinylidene fluoride (PVDF) membrane (Millipore, Bedford, MA, USA). The membrane was blocked with Western washing buffer (TBST) containing $5 \% \mathrm{BSA}$ and then incubated with an appropriate concentration of primary antibody at $4{ }^{\circ} \mathrm{C}$ overnight. Glyceraldehyde-3-phosphate dehydrogenase (GAPDH) protein was used as an internal control. The main antibodies used in the WB analysis included the following: anti-Ncadherin (13116S, Cell Signaling Technology, Beverley, MA, 
USA), anti-E-cadherin (3195S, Cell Signaling Technology, Beverley, MA, USA), anti-vimentin (5741S, Cell Signaling Technology, Beverley, MA, USA), anti-GAPDH (5174T, Cell Signaling Technology, Beverley, MA, USA), anti-Bcl-2 (4223S, Cell Signaling Technology, Beverley, MA, USA), anti-Bax (5023S, Cell Signaling Technology, Beverley, MA, USA), anti-matrix metalloproteinase 2 (MMP-2) (40993S, Cell Signaling Technology, Beverley, MA, USA), antiMMP-9 (3852S, Cell Signaling Technology, Beverley, MA, USA), anti-JAK2 (3230S, Cell Signaling Technology, Beverley, MA, USA), anti-p-STAT3 (9145S, Cell Signaling Technology, Beverley, MA, USA), and anti-STAT3 (12640S, Cell Signaling Technology, Beverley, MA, USA).

The next day, the membrane was washed three times with TBST and then incubated with an appropriate horseradish peroxidase (HRP)-conjugated secondary membrane antibody (ab6721, Abcam, Cambridge, MA, USA) at room temperature for 2 hours. After washing three times with TBST, the protein bands were observed using enhanced chemiluminescence (ECL) substrate (Millipore) and scanned using the Tanon 4200 chemiluminescent image analysis system (Tanon, Shanghai, China). This experiment was repeated three times.

\section{Cell immunofluorescence}

A549 cells were inoculated into a six-well plate using a cellclimbing method. After 12 hours, 25, 50, and $100 \mu \mathrm{M}$ PC solutions were added, and only $0.1 \%$ DMSO was added as the control group. The cells were then incubated for 12 hours at $37{ }^{\circ} \mathrm{C}, 5 \% \mathrm{CO}_{2}$. Next, the cells were fixed with ice-cold methanol, and the membrane was permeated with $0.5 \%$ Triton-X in PBS, blocked in 5\% BSA solution, and incubated p-STAT3 primary antibody (Cell Signal Technology, Danvers, MA, USA) at $4{ }^{\circ} \mathrm{C}$ (dilution ratio $1: 100)$. After washing off the primary antibody the next day, the cells were incubated with the fluorescent secondary antibody (Abcam, Cambridge, UK) (dilution ratio 1:400). Finally, the nuclei were stained with 4',6-diamidino-2phenylindole (DAPI), and images were taken using an FV1000 confocal microscope (Olympus, Tokyo, Japan). This experiment was repeated three times. Effect of $\mathcal{7 A 2} / S T A T 3$ signal pathway inhibitor, AG490, on
the proliferation, invasion, and migration of $A 549$ cells

A549 cells were treated with $0.1 \%$ DMSO (control group), procyanidins $50 \mu \mathrm{M}$ (procyanidins group), and procyanidins $50 \mu \mathrm{M}+\mathrm{AG} 49025 \mu \mathrm{M}$ (AG490 + procyanidins group) The wound-healing assay and the Transwell migration and invasion assays were used to detect PCs' migration and invasion effects on A549 cells. The cell growth assay was used to detect the anti-cell growth ability of PCs on A549 cells. WB was used to detect the expression levels of JAK2 and p-STAT3.

\section{Statistical analysis}

Data were expressed as the mean \pm standard deviation (SD) of three independent experiments. The Student's $t$-test or one-way analysis of variance in GraphPad Pro 7.0 (GraphPad, San Diego, CA) analyzed the statistical differences between different groups. $\mathrm{P}$ values less than 0.05 $(\mathrm{P}<0.05)$ were considered to be indicative of significance.

\section{Ethical statement}

The study was conducted in accordance with the Declaration of Helsinki (as revised in 2013).

\section{Results}

\section{PCs reduce the proliferation and cloning ability of $A 549$ cells}

A549 cells were treated with $0,12.5,25,50,100,200 \mu \mathrm{M}$ procyanidins for 24 and 48 hours (Figure 1A). The results of absorbance measured by the Synergy H1 microplate reader at 24 hours showed the following: $0.853 \pm 0.107$, $0.748 \pm 0.099,0.628 \pm 0.052,0.521 \pm 0.067,0.501 \pm 0.117$; and the half maximal inhibitory concentration (IC50) of PCs on A549 cells was $150.7 \mu \mathrm{M}$ (Figure 1B). Furthermore, the absorbance (measured by the Synergy H1 microplate reader) at 48 hours showed the following: $0.760 \pm 0.040$, $0.701 \pm 0.075,0.629 \pm 0.056,0.415 \pm 0.093,0.345 \pm 0.050$; and the IC50 of PCs on A549 cells was $78.41 \mu \mathrm{M}$ (Figure 1B). These results suggested that PCs significantly inhibited the growth and proliferation of human A549 cells in a concentration-dependent manner.

PC effect on the cloning ability of A549 was observed using a clonal formation assay (Figure 1C,D). After 0, 25, 50, $100 \mu \mathrm{M}$ procyanidins were applied to A549 cells for 1 week, the number of colonies was observed after staining with crystal violet. As the PC concentration increased, the amount of colony formation decreased. This shows that 
A<smiles>Oc1cc(O)c2c(c1)OC(c1ccc(O)c(O)c1)C(OC1(c3ccc(O)c(O)c3)Oc3cc(O)cc(O)c3C(O)C1O)C2</smiles>

Chemical structure of Proantho Cyanidins (PC)
B

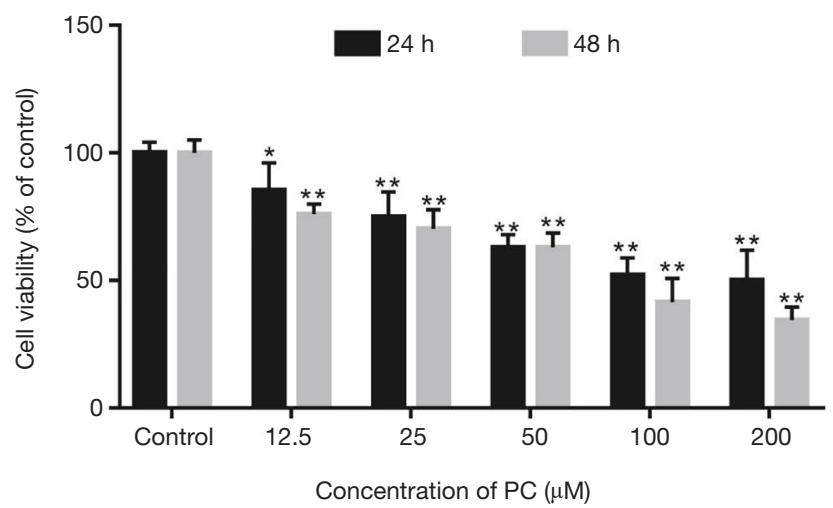

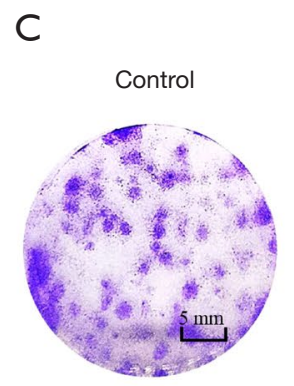
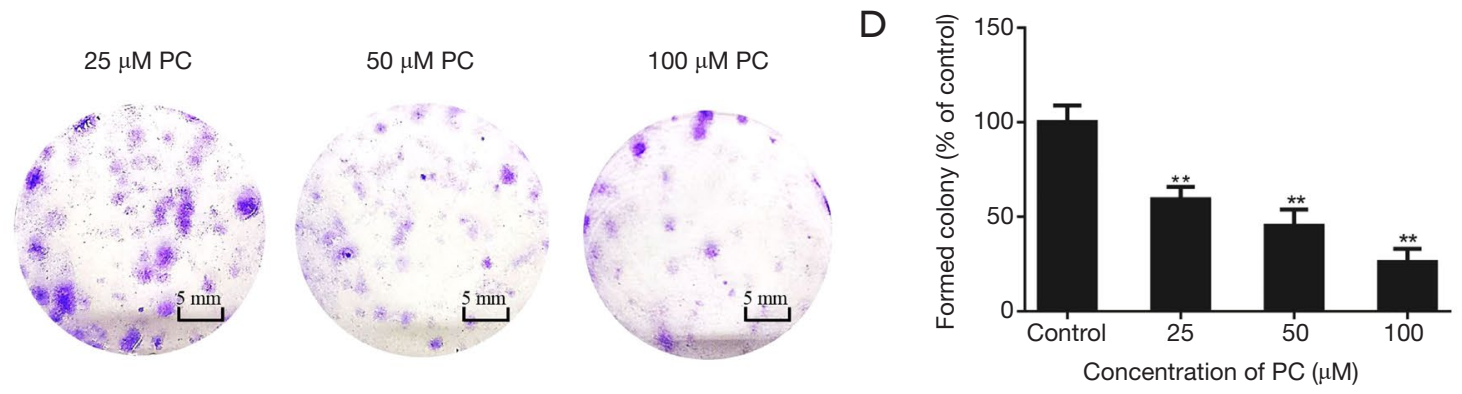

Figure 1 Chemical structure of proanthocyanidins (PCs) and the effects of PC on the cell growth of A549 cells. (A) Chemical structure of PCs. (B) Cell viability: A549 cell lines were treated with various PC concentrations for 24/48 hours and analyzed using a cell growth assay. (C) Colony formation: A549 cells were treated with a PC concentration gradient and incubated for 1 week. The clones were then fixed with 4\% paraformaldehyde and stained with crystal violet, and PC was found to inhibit the colony formation of A549 cells. (D) Representative histogram of the colony formation analysis in A549 cells treated with various concentrations of $\mathrm{PC}$. *, $\mathrm{P}<0.05 ;{ }^{* *}, \mathrm{P}<0.01$.

PCs reduced the cloning ability of A549.

\section{PCs significantly inhibit the migration and invasion of A549 cells}

The microscopic observation of the wound healing assay showed that different procyanidins concentrations inhibited the migration of A549 cells (Figure $2 A, B$ ). After 24 hours of scratches, the scratches became narrower, and A549 cells migrated to the inside of the scratches. With the increase in $\mathrm{PC}$ concentration, the migration ability of A549 cells decreased more obviously. This shows that PCs can effectively inhibit the migration speed of A549 cells. Migration and invasion assays results showed that after treatment with $25,50,100 \mu \mathrm{M}$ PC, the number of transmembrane cells decreased significantly $(\mathrm{P}<0.05)$ (Figure $2 C, D, E, F)$, indicating that PCs can inhibit the migration and invasion of A549 cells. These experimental results show that PCs can inhibit the migration and invasion of NSCLC A549 cells.

\section{PC induces G2/M cell cycle arrest in A549 cells}

To investigate the PC effect on the A549 cell cycle, the cells were treated with $0,25,50,100 \mu \mathrm{M}$ PCs for 24 hours, and then the cell cycle was detected by flow cytometry. As shown in Figure 3, PCs induced G2/M phase arrest in A549 cells in a concentration-dependent manner. As the concentration of PCs increased, the effect of inducing G2/M phase arrest in A549 cells was more significant.

\section{PC induces apoptosis in A549 cells}

Quantitative analysis of annexin V-PE/7-ADD staining by flow cytometry showed that the total apoptosis score was $5.14 \%$ in the control group, $16.15 \%$ in the $25 \mu \mathrm{M}$ group, $24.83 \%$ in the $50 \mu \mathrm{M}$ group, and $31.5 \%$ in the $100 \mu \mathrm{M}$ group (Figure $4 A, B$ ). PC-induced down-regulation of the anti-apoptotic protein, $\mathrm{Bcl}-2$, and up-regulation of the pro-apoptotic protein, Bax, was observed in A549 cells (Figure $4 C$ ). This indicates that PCs induce apoptosis in 
A
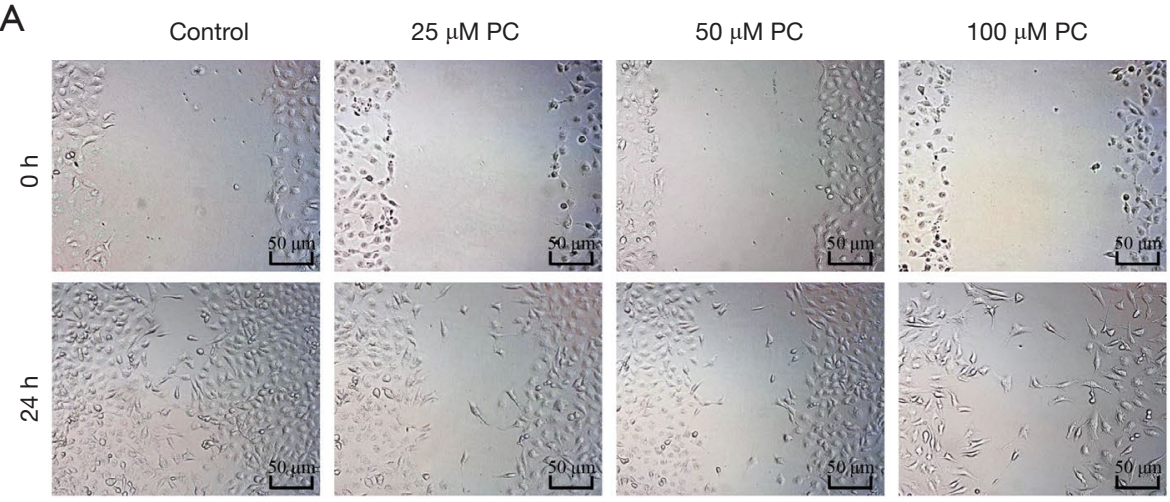

C

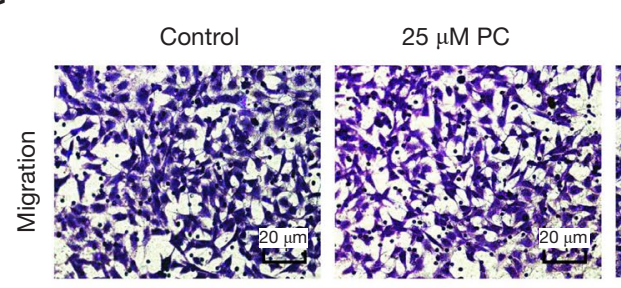

$50 \mu \mathrm{M}$ PC

$100 \mu \mathrm{M} P \mathrm{PC}$
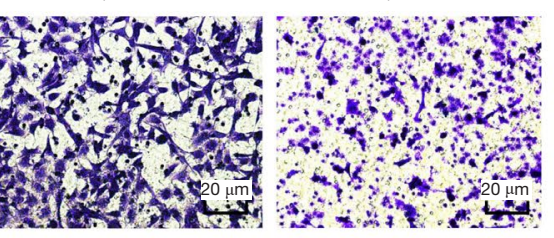

$E$

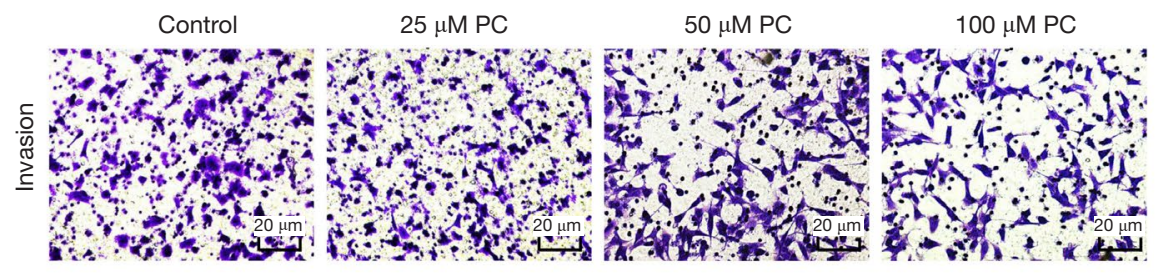

B
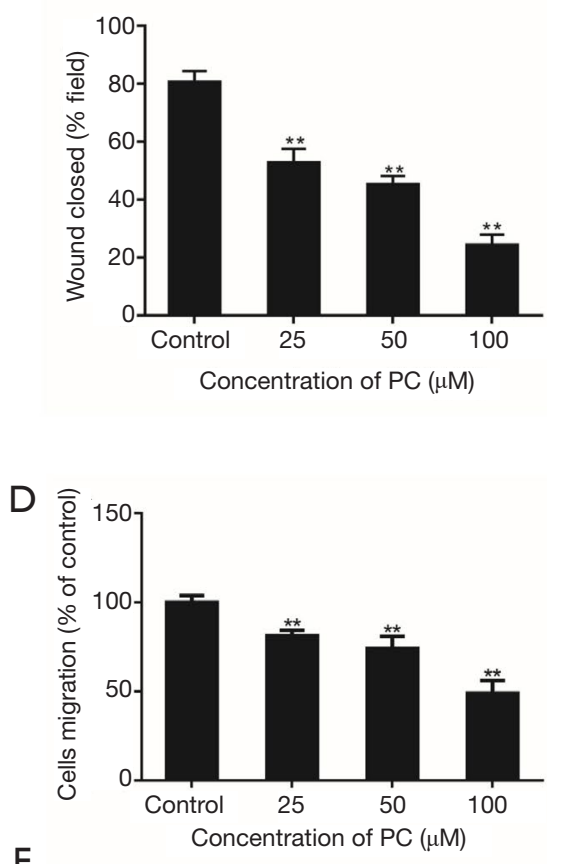

$\mathrm{F}$

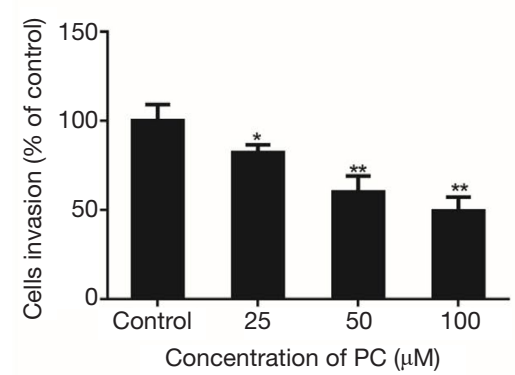

Figure 2 Effects of PC on migration and invasion of A549 cell lines after treatment with a proanthocyanidin (PC) concentration gradient. (A) Wound closure time-course of A549 cell subpopulations after scratching and treatment with various concentrations of PC. Optical microscope observed A549 cell subpopulations. Magnification, $\times 100$. (B) Representative histogram of the wound closure analysis in A549 cells treated with various concentrations of PC. (C) The number of migrating cells was significantly decreased after PC treatment. Magnification, $\times 200$. The cells were fixed with $4 \%$ paraformaldehyde and stained with crystal violet. (D) Representative histogram of cell migration in A549 cells treated with various concentrations of PC. (E) The number of invading cells was significantly decreased after PC treatment. Magnification, $\times 200$. The cells were fixed with $4 \%$ paraformaldehyde and stained with crystal violet. $(\mathrm{F})$ Representative histogram of cell invasion in A549 cells treated with various concentrations of $\mathrm{PC} .{ }^{*}, \mathrm{P}<0.05 ;{ }^{* *}, \mathrm{P}<0.01$.

A549 cells.

\section{PCs inbibit the FAK2/STAT3 pathway}

WB was used to detect the protein expression levels of JAK2, p-STAT3, STAT3, MMP-2, and MMP-9. Compared with the control group $(\mathrm{PC}=0 \mu \mathrm{M})$, the expression of JAK2 and p-STAT3 in the experimental group $(\mathrm{PCs}=25$,
50, and $100 \mu \mathrm{M}$, respectively) was reduced, the expression of STAT3 was basically unchanged, and the expression of the MMP-2 and MMP-9 proteins was down-regulated (Figure 5). Moreover, the changes were more obvious in the 50 and $100 \mu \mathrm{M}$ groups, and the differences were statistically significant. The cell immunofluorescence results showed that the expression of the p-STAT3 protein in the control group was obvious. As the PC concentration increased, the 

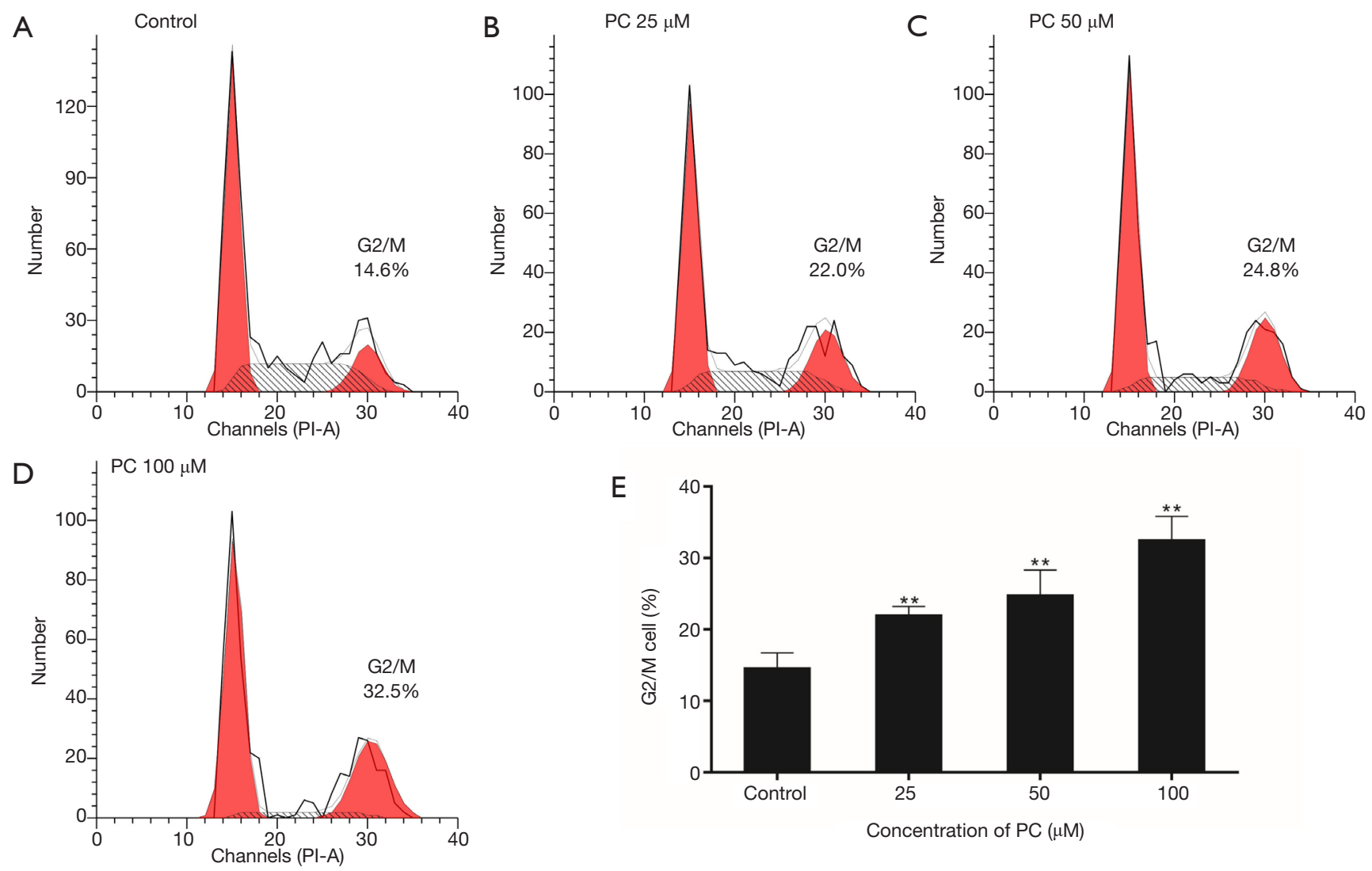

Figure 3 PC induces G2/M cell cycle arrest in A549 cells. (A,B,C,D) Induction of cell cycle arrest in A549 cells was analyzed by flow cytometry after treatment with a proanthocyanidin (PC) concentration gradient for 24 hours. (E) Representative histogram of the flow cytometry analysis in A549 cells treated with various concentrations of PC. Assays were performed in triplicate. ${ }^{* *}, \mathrm{P}<0.01$.

expression of the p-STAT3 protein was down-regulated in a concentration-dependent manner (Figure 6).

\section{PCs inbibit EMT}

WB was also used to detect the protein expressions of E-cadherin, vimentin, and $\beta$-catenin. Compared with the control group $(\mathrm{PC}=0 \mu \mathrm{M})$, the expression of E-cadherin in the experimental group ( $\mathrm{PCs}=25,50$, and $100 \mu \mathrm{M}$, respectively) was significantly increased, and the expression of $\mathrm{N}$-cadherin and vimentin were down-regulated (Figure 5).

\section{AK2/STAT3 signal patbway inbibitor AG490 cooperates with PCs to inbibit A549 cell invasion and migration and induce apoptosis}

Compared with the other groups, the expressions of JAK2 and p-STAT3 were markedly decreased in the expressions in the PCs + AG490 group (Figure 7A). The cell growth assay results (Figure $7 B$ ) showed that the proliferation of A549 cells in the PCs + AG490 group was significantly weaker than the other groups. The wound-healing assay and migration and invasion assays results (Figure $7 C, D, E, F, G, H$ ) showed that cell migration and invasion were notably inhibited in the PCs + AG490 group compared with the other groups. These results indicated that AG490 cooperates with PCs to inhibit A549 cell invasion and migration and induce apoptosis.

\section{Discussion}

Lung cancer is a malignant tumor and is the leading cause of cancer-related morbidity and mortality worldwide. The number of lung cancer deaths globally exceeds 1.56 million every year $(11,12)$. NSCLC accounts for about $85 \%$ of all lung cancers (1). Despite the progress made in lung cancer treatment in recent years, including targeted molecular therapy and immunotherapy, the 5-year survival 

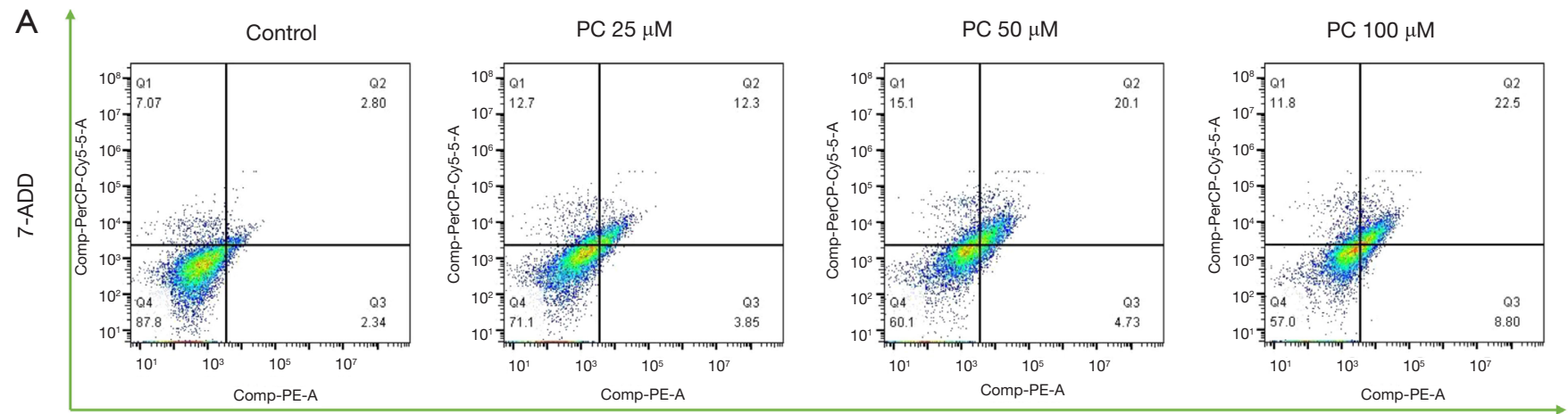

B

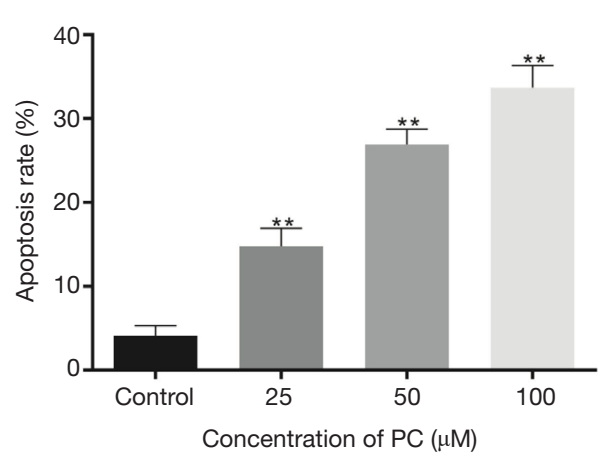

Annexin V

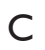

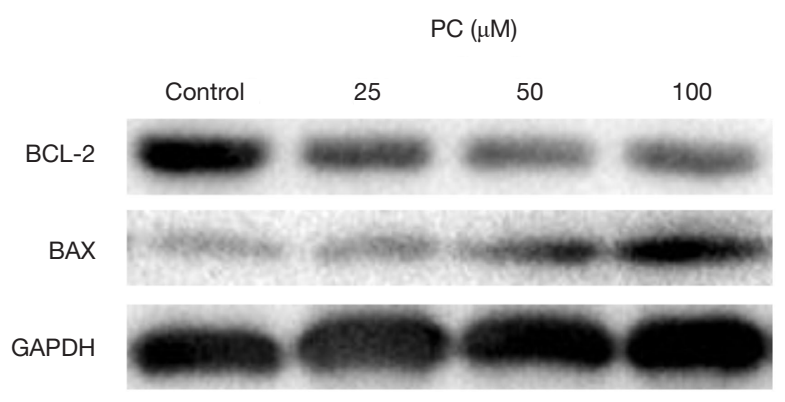

Figure 4 Proanthocyanidin (PC) promoted A549 cell apoptosis. (A) The induction of apoptosis in A549 cells was analyzed by flow cytometry after treatment with a PC concentration gradient. (B) Representative histogram of the flow cytometry analysis in A549 cells treated with various concentrations of PC. Assays were performed in triplicate. (C) Western blotting analysis of apoptosis-related proteins. The expression of Bcl-2 and Bax in A549 cells. **, $\mathrm{P}<0.01$.

rate of NSCLC remains very low, with only $17.7 \%$ of lung cancer patients surviving for more than 5 years after diagnosis $(2,13)$.

PCs are a class of polyphenolic polymers with flavanols and their derivatives as structural units and are widely distributed in the plant kingdom $(14,15)$. PCs are highly effective antioxidants. Grape seed PCs exhibit a wide range of functions and uses, including scavenging free radicals, regulating blood lipids, anti-inflammatory effects, etc. $(5,6,16)$. In recent years, it has also been found that PCs have antitumor effects on various tumors, including bladder and cervical cancers, head and neck squamous cell carcinoma, glioma, melanoma, and liver cancer (17-21).

At present, there are only a few reports on the effects of PCs on lung cancer. Some studies have found that PCs inhibit lung cancer by promoting apoptosis (18); however, few studies explore the effect of procyanidins on lung cancer invasion, migration, and proliferation. Also, relevant reports on the antitumor mechanism of PCs on lung cancer are lacking.

The JAK protein family is a class of non-receptor tyrosine kinases, and STAT3 is its downstream signaling protein, which JAK family enzymes can activate to regulate cytokine and growth factor signaling. The JAK2/STAT3 signaling pathway is mainly involved in cell proliferation, differentiation, apoptosis, and immune regulation (22). In normal tissues, the JAK2/STAT3 signaling pathway is tightly regulated. STAT is in an inactive state; however, it is abnormally activated and highly expressed in tumor cells (23). The activation of the JAK2/STAT3 pathway can regulate the occurrence and development of various cancers and promote cancer infiltration, metastasis, and EMT $(24,25)$. The JAK2/STAT3 signaling pathway also plays an important role in the occurrence and development of lung cancer (26-28).

Metastatic ability is a major feature of malignant tumors and is a key reason for its difficulty. Migration and invasiveness can reflect the metastatic ability of tumors. In this study, we found that through wound healing and migration and invasion assays, PCs can invade and migrate into lung cancer A549 cells, and all have a strong inhibitory effect. The cell growth and clonal formation assays showed 
$\mathrm{PC}(\mu \mathrm{M})$

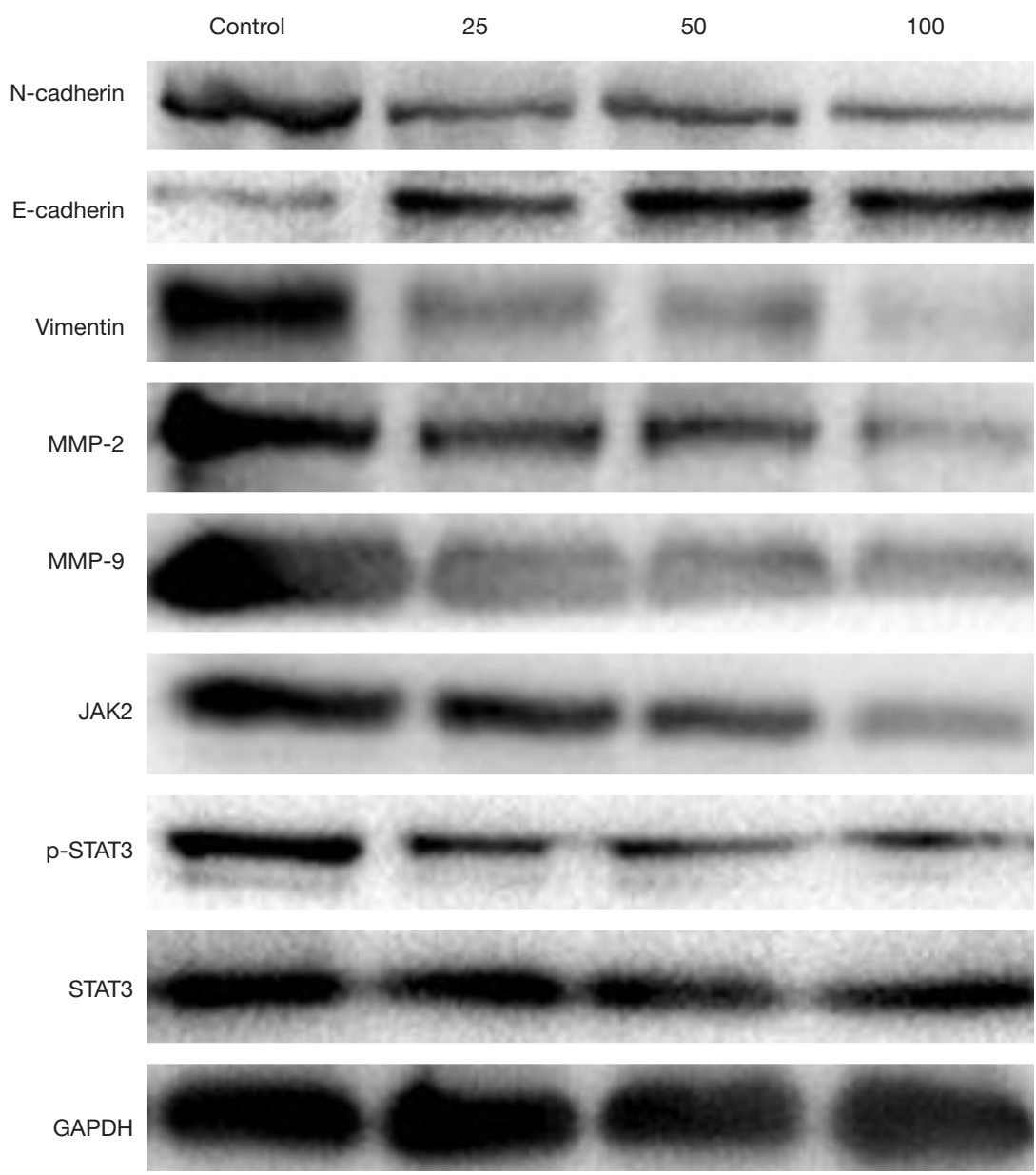

Figure 5 Western blot analysis of MMP-2, MMP-9, JAK2, p-STAT3, and STAT3 in A549 cells. MMP, matrix metalloproteinase; JAK2, Janus kinase 2; STAT3, signal transducer and activator of transcription 3.

that PCs have an inhibitory effect on the proliferation and cloning ability of A549 cells. In this study, flow cytometry was used to detect the cycle distribution of A549 cells. We found that the number of cells in the G2/M phase increased, and the proportion of cells increased significantly. This cell cycle arrest was dose-dependent concerning drug concentration.

An important mechanism of action of anticancer drugs is the induction of tumor cell apoptosis (29). Typically, apoptosis maintains the internal environment's stability. Genes control the autonomous and orderly cell death process, which is also known as programmed death. If apoptosis is blocked, the rate of abnormal cell proliferation will exceed the rate of apoptosis and accelerate the formation of malignant tumors (30). Therefore, inducing tumor cell apoptosis has become an important role of anticancer drugs. We observed the effect of PCs on the apoptosis of A549 lung cancer cells. The flow cytometry results showed that with the increase in PC concentration, the ratio of early and late apoptosis of A549 cells cocultured with PCs increased, suggesting that PCs can induce the apoptosis of A549 cells.

EMT is closely related to tumor invasion, migration, and metastasis (31). WB results showed that, compared with the control group, E-cadherin expression was significantly increased in the combination experimental group, whereas the expression of $\mathrm{N}$-cadherin and vimentin was notably decreased (Figure 5). This indicates that PCs can 
Control
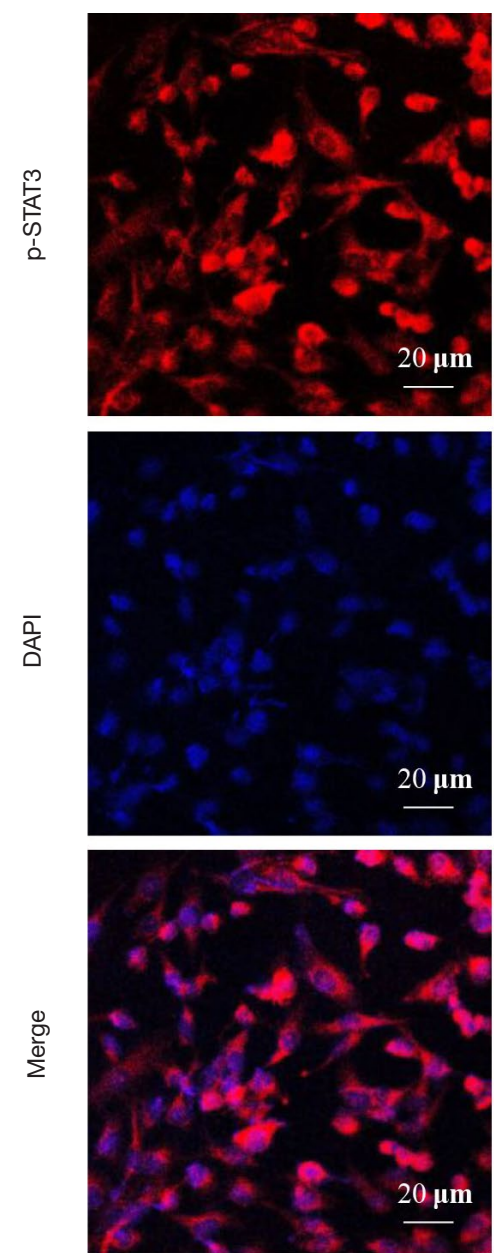

25
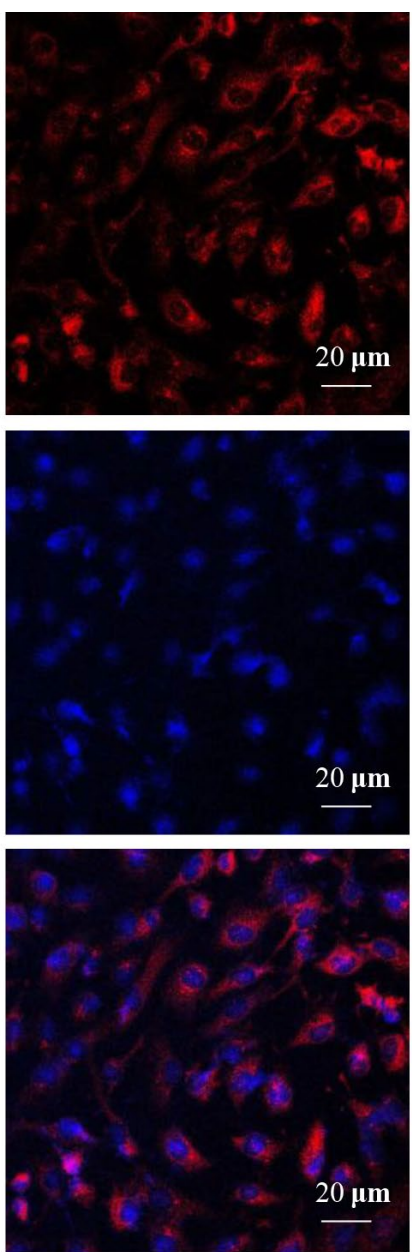

50
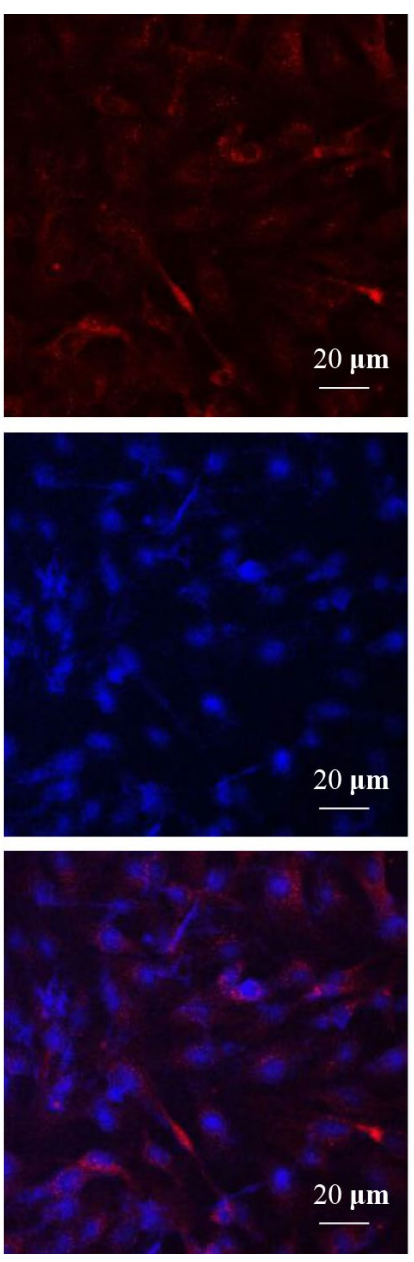

100
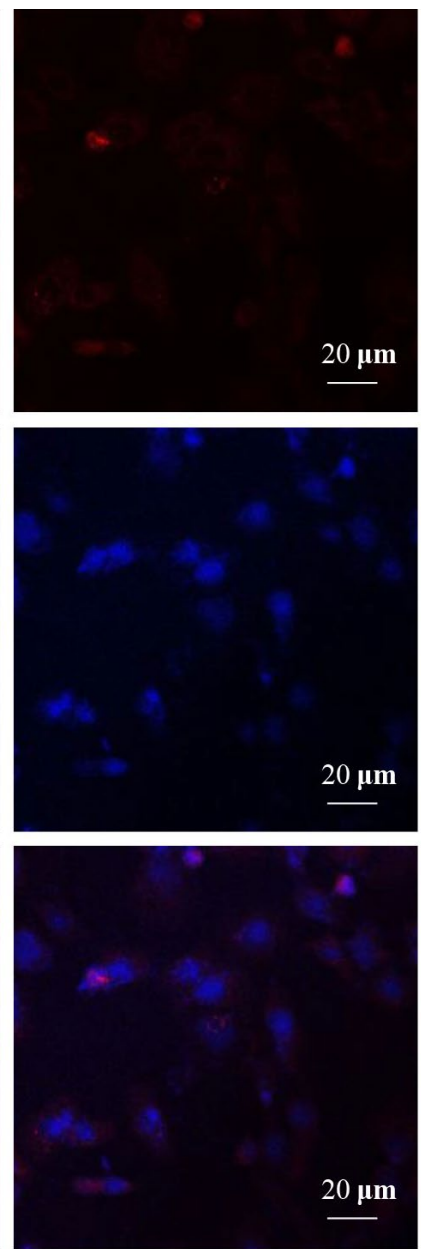

Figure 6 Cell immunofluorescence results. The expression of the p-STAT3 protein in the control group was obvious. As the concentration of procyanidin increased, the expression of the p-STAT3 protein was down-regulated in a concentration-dependent manner. Red: staining of p-STAT3 protein; Blue: staining of cell nuclei by DAPI.

significantly inhibit the EMT of A549 cells.

To explore the possible anti-lung cancer mechanism of PCs, we used cell immunofluorescence and WB to detect the changes in the expression of the JAK2/STAT3 signaling pathway. We found that the STAT3 protein expression remained unchanged, while the JAK2 and p-STAT3 protein expressions declined. Additionally, we found that JAK2/ STAT3 signaling pathway inhibitors could cooperate with PCs to inhibit the occurrence and development of lung cancer A549 cells, suggesting that PCs can play an anti-lung cancer effect through the STAT3 signaling pathway.

In summary, PCs can inhibit NSCLC A549 cell proliferation, invasion, metastasis, clone formation, EMT, and induced apoptosis and G2/M cell cycle arrest. PCs work by inhibiting the JAK2/STAT3 signaling pathway. As a novel antitumor drug, PCs have broad application prospects for the treatment of NSCLC. The next step is to conduct animal experiments to clarify the antitumor effect of PCs in vivo. Also, the pharmacokinetics, pharmacodynamics, and toxicity of PCs need to be comprehensively evaluated. We also plan to study the combined application of PCs and other clinical anticancer drugs to improve PCs' antitumor effect and verify their effect on NSCLC. We believe that PCs can be used clinically better to treat lung cancer 
A

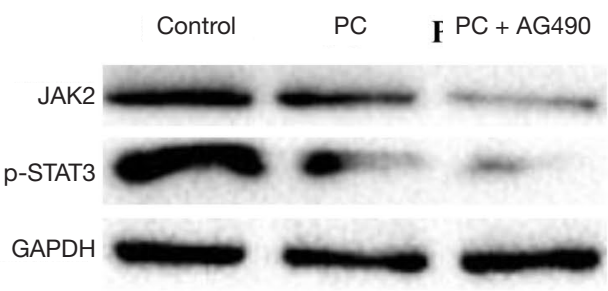

C

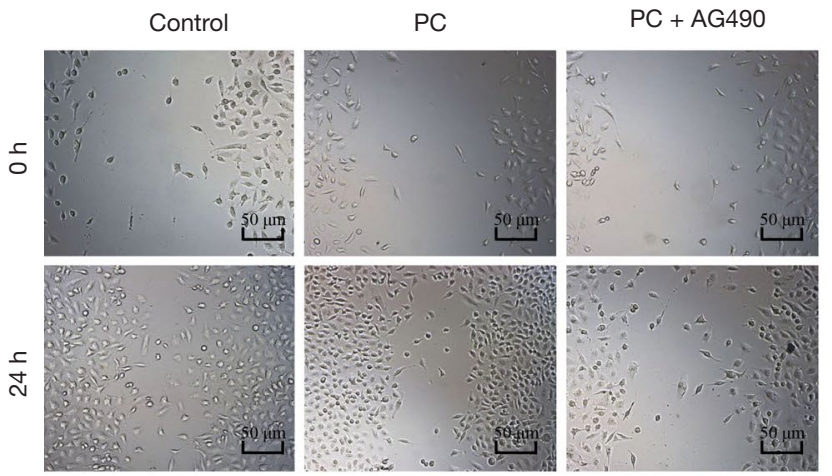

E

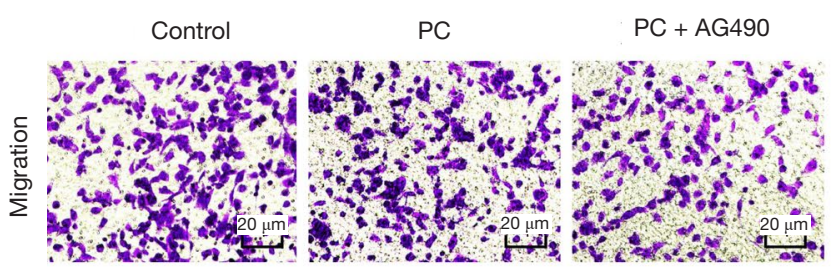

G

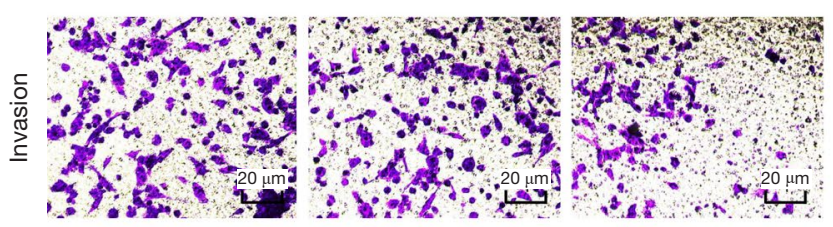

B

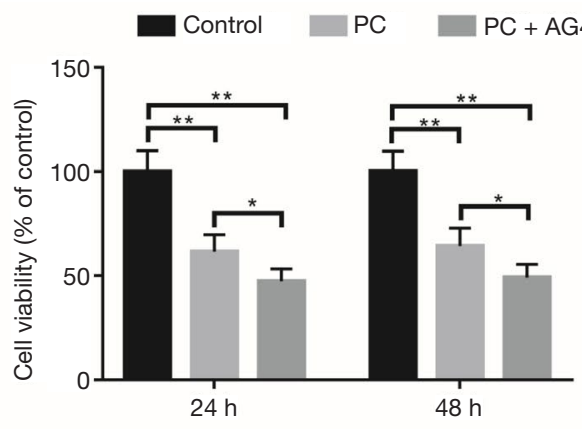

D

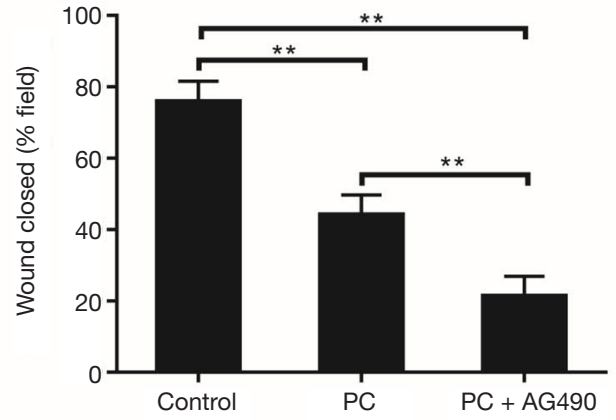

F

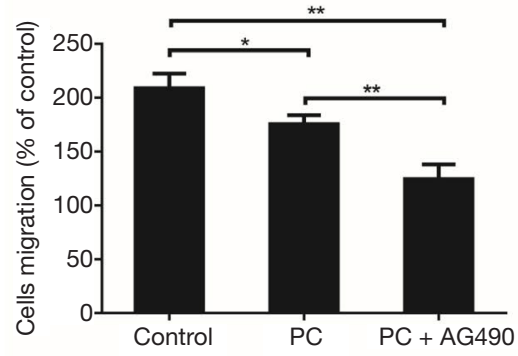

$\mathrm{H}$

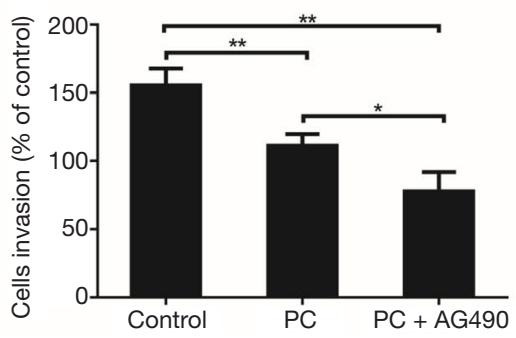

Figure 7 Effects of proanthocyanidin (PC) and AG490 on the JAK2/STAT3 pathway and the migration and invasion of A549 cells after treatment with PC or AG490 + PC. (A) Western blotting analysis of JAK2 and p-STAT3 in A549 cells. (B) Cell viability: A549 cell lines were treated with PC or AG490 + PC for 24/48 hours and were analyzed using a cell growth assay (the IC50 values were calculated). (C,D) Wound closure time-course of the A549 cell subpopulations treated with PC or AG490 + PC after scratching. Optical microscope observed A549 cell subpopulations. Magnification, $\times 100$. (E,F) The number of migrating cells was significantly decreased after PC or AG490 + PC treatment. Magnification, $\times 200$. The cells were fixed with $4 \%$ paraformaldehyde and stained with crystal violet. $(G, H)$ The number of invading cells was significantly decreased after PC or AG490 + PC treatment. Magnification, $\times 200$. The cells were fixed with $4 \%$ paraformaldehyde and stained with crystal violet. *, $\mathrm{P}<0.05$; ${ }^{* *}, \mathrm{P}<0.01$. JAK2, Janus kinase 2; STAT3, signal transducer and activator of transcription 3. 
patients shortly.

\section{Acknowledgments}

Funding: This study was supported by grants from the Young Scientists Fund of the National Natural Science Foundation of China (No. 81702252).

\section{Footnote}

Reporting Checklist: The authors have completed the MDAR reporting checklist. Available at http://dx.doi.org/10.21037/ tcr-20-3018

Data Sharing Statement: Available at http://dx.doi. org/10.21037/tcr-20-3018

Conflicts of Interest: All authors have completed the ICMJE uniform disclosure form (available at http://dx.doi. org/10.21037/tcr-20-3018). The authors have no conflicts of interest to declare.

Ethical Statement: The authors are accountable for all aspects of the work in ensuring that questions related to the accuracy or integrity of any part of the work are appropriately investigated and resolved. This study was conducted in accordance with the Declaration of Helsinki (as revised in 2013).

Open Access Statement: This is an Open Access article distributed in accordance with the Creative Commons Attribution-NonCommercial-NoDerivs 4.0 International License (CC BY-NC-ND 4.0), which permits the noncommercial replication and distribution of the article with the strict proviso that no changes or edits are made and the original work is properly cited (including links to both the formal publication through the relevant DOI and the license). See: https://creativecommons.org/licenses/by-nc-nd/4.0/.

\section{References}

1. Siegel RL, Miller KD, Jemal A. Cancer statistics, 2019. CA Cancer J Clin 2019;69:7-34.

2. Yatabe Y, Kerr KM, Utomo A, et al. EGFR mutation testing practices within the Asia Pacific region: results of a multicenter diagnostic survey. J Thorac Oncol 2015;10:438-45.

3. Fine AM. Oligomeric proanthocyanidin complexes: history, structure, and phytopharmaceutical applications. Altern Med Rev 2000;5:144-51.

4. Dai J, Wang G, Li W, et al. High-throughput screening for anti-influenza $\mathrm{A}$ virus drugs and study of the mechanism of procyanidin on influenza A virus-induced autophagy. J Biomol Screen 2012;17:605-17.

5. Wu S, Yue Y, Li J, et al. Procyanidin B2 attenuates neurological deficits and blood-brain barrier disruption in a rat model of cerebral ischemia. Mol Nutr Food Res 2015;59:1930-41.

6. Zhang H, Sun XQ, Cao JM, et al. Protective effect of epimedium combined with oligomeric proanthocyanidins on exercise-induced renal ischemia-reperfusion injury of rats. Int J Clin Exp Med 2014;7:5730-6.

7. Chen L, Yan F, Chen W, et al. Procyanidin from peanut skin induces antiproliferative effect in human prostate carcinoma cells DU145. Chem Biol Interact 2018;288:12-23.

8. Connor CA, Adriaens M, Pierini R, et al. Procyanidin induces apoptosis of esophageal adenocarcinoma cells via JNK activation of c-Jun. Nutr Cancer 2014;66:335-41.

9. Mao JT, Lu QY, Xue B, et al. A Pilot Study of a Grape Seed Procyanidin Extract for Lung Cancer Chemoprevention. Cancer Prev Res (Phila) 2019;12:557-66.

10. Xue B, Lu QY, Massie L, et al. Grape seed procyanidin extract against lung cancer: the role of microrna106b, bioavailability, and bioactivity. Oncotarget 2018;9:15579-90.

11. Torre LA, Bray F, Siegel RL, et al. Global cancer statistics, 2012. CA Cancer J Clin 2015;65:87-108.

12. Ferlay J, Soerjomataram I, Dikshit R, et al. Cancer incidence and mortality worldwide: sources, methods and major patterns in GLOBOCAN 2012. Int J Cancer 2015;136:E359-86.

13. Pietanza MC, Byers LA, Minna JD, et al. Small cell lung cancer: will recent progress lead to improved outcomes? Clin Cancer Res 2015;21:2244-55.

14. Naczk M, Shahidi F. Extraction and analysis of phenolics in food. J Chromatogr A 2004;1054:95-111.

15. Yang Y, Chien M. Characterization of grape procyanidins using high-performance liquid Chromatography/Mass spectrometry and matrix-assisted laser Desorption/ Ionization time-of-flight mass spectrometry. J Agric Food Chem 2000;48:3990-6.

16. Hamidian Jahromi A, Roozbeh J, Bastani B. Potential protective effect of grape seed proanthocyanidine extract in cold ischemia-reperfusion injury of the transplanted kidney. Iran J Kidney Dis 2013;7:327-8. 
17. Bao L, Cai X, Dai X, et al. Grape seed proanthocyanidin extracts ameliorate podocyte injury by activating peroxisome proliferator-activated receptor- $\gamma$ coactivator $1 \alpha$ in low-dose streptozotocin-and high-carbohydrate/highfat diet-induced diabetic rats. Food Funct 2014;5:1872-80.

18. Fernández-Iglesias A, Quesada H, Díaz S, et al. Combination of grape seed proanthocyanidin extract and docosahexaenoic acid-rich oil increases the hepatic detoxification by GST mediated GSH conjugation in a lipidic postprandial state. Food Chem 2014;165:14-20.

19. Ohnuma T, Anzai E, Suzuki Y, et al. Selective antagonization of activated Nrf2 and inhibition of cancer cell proliferation by procyanidins from Cinnamomi Cortex extract. Arch Biochem Biophys 2015;585:17-24.

20. Kresty LA, Weh KM, Zeyzus-Johns B, et al. Cranberry proanthocyanidins inhibit esophageal adenocarcinoma in vitro and in vivo through pleiotropic cell death induction and PI3K/AKT/mTOR inactivation. Oncotarget 2015;6:33438-55.

21. Bishayee A, Mbimba T, Thoppil RJ, et al. Anthocyaninrich black currant (Ribes nigrum L.) extract affords chemoprevention against diethylnitrosamine-induced hepatocellular carcinogenesis in rats. J Nutr Biochem 2011;22:1035-46.

22. Liu RY, Zeng Y, Lei Z, et al. JAK/STAT3 signaling is required for TGF- $\beta$-induced epithelial-mesenchymal transition in lung cancer cells. Int J Oncol 2014;44:1643-51.

23. Kowshik J, Baba AB, Giri H, et al. Astaxanthin inhibits JAK/STAT-3 signaling to abrogate cell proliferation, invasion and angiogenesis in a hamster model of oral

Cite this article as: $\mathrm{Wu} \mathrm{Y,} \mathrm{Liu} \mathrm{C,} \mathrm{Niu} \mathrm{Y,} \mathrm{Xia} \mathrm{J,} \mathrm{Fan} \mathrm{L,} \mathrm{Wu} \mathrm{Y,}$ Gao W. Procyanidins mediates antineoplastic effects against non-small cell lung cancer via the JAK2/STAT3 pathway. Transl Cancer Res 2021;10(5):2023-2035. doi: 10.21037/tcr-20-3018 cancer. PloS One 2014;9:e109114.

24. Teng Y, Ross JL, Cowell JK. The involvement of JAKSTAT3 in cell motility, invasion, and metastasis. JAKSTAT 2014;3:e28086.

25. Sun D, Shen $W$, Zhang F, et al. $\alpha$-Hederin inhibits interleukin 6-induced epithelial-to-mesenchymal transition associated with disruption of JAK2/STAT3 signaling in colon cancer cells. Biomed Pharmacother 2018;101:107-14.

26. Li R, Huang Y, Lin J. Distinct effects of general anesthetics on lung metastasis mediated by IL-6/JAK/STAT3 pathway in mouse models. Nat Commun 2020;11:642.

27. Zhang T, Ma L, Wu P, et al. Gallic acid has anticancer activity and enhances the anticancer effects of cisplatin in non-small cell lung cancer A549 cells via the JAK/STAT3 signaling pathway. Oncol Rep 2019;41:1779-88.

28. Lin XL, Liu M, Liu Y, et al. Transforming growth factor $\beta 1$ promotes migration and invasion in HepG2 cells: Epithelial-to-mesenchymal transition via JAK/STAT3 signaling. Int J Mol Med 2018;41:129-36.

29. Bachawal SV, Wali VB, Sylvester PW. Enhanced antiproliferative and apoptotic response to combined treatment of gamma-tocotrienol with erlotinib or gefitinib in mammary tumor cells. BMC cancer 2010;10:84.

30. Li T, Kon N, Jiang L, et al. Tumor suppression in the absence of p53-mediated cell-cycle arrest, apoptosis, and senescence. Cell 2012;149:1269-83.

31. Yang S, Liu Y, Li MY, et al. FOXP3 promotes tumor growth and metastasis by activating $W n t / \beta$-catenin signaling pathway and EMT in non-small cell lung cancer. Mol Cancer 2017;16:124. 